\title{
Una nueva especie de Ophryosporus (Eupatorieae: Asteraceae) para el Perú
}

\author{
New species of Ophryosporus (Eupatorieae Asteraceae) from Peru
}

Abundio Sagástegui Alva y Eric F. Rodríguez Rodríguez

\begin{abstract}
Herbarium Truxillense (HUT), Universidad Nacional de Trujillo. Jr. San Martín 392. Trujillo, Perú. E-mail Abundio Sagástegui: abundiosag@hotmail.com E-mail Eric F. Rodríguez: efr@@unitru.edu.pe
\end{abstract}

\section{Resumen}

Se describe e ilustra una nueva especie de Ophryosporus Meyen (Eupatorieae: Asteraceae) procedente del Departamento de Cajamarca, Perú, y aparentemente endémica a la Provincia de Contumazá, denominada Ophryosporus marchii Sagást. \& E. Rodr. Esta nueva especie está relacionada con la especie O. sagasteguii $\mathrm{H}$. Rob. con la cual se discute críticamente. Adicionalmente se presentan datos sobre su distribución geográfica y ecológica, así como su estado de conservación.

Palabras clave: Ophryosporus marchii, Asteraceae, nueva especie, Contumazá, Cajamarca, Perú.

\section{Abstract}

Ophryosporus marchii Sagást. \& E. Rodr. is described as a new species of Ophryosporus Meyen (Eupatorieae: Asteraceae) from the Department of Cajamarca, Peru. This new species is apparently endemic to the province of Contumaza and closest relative to $\mathrm{O}$. sagasteguii $\mathrm{H}$. Rob. It is critically compared with this species and data on its geographical distribution, ecology and conservation status are provided.

Presentado: $\quad 24 / 10 / 2007$ Aceptado: $\quad 11 / 01 / 2008$

\section{Introducción}

El género americano Ophryosporus (Eupatorieae: Asteraceae) descrito por Meyen en 1827, presenta alrededor de 48 especies, siendo la mayoría de Sudamérica. Para la flora del Perú se han registrado 21 especies (Brako \& Zarucchi, 1993; Ulloa Ulloa et al., 2004), de ellas 11 son endémicas a saber: Ophryosporus apricus B.L. Rob., O. bipinnatifidus B.L. Rob., O. ferreyrii H. Rob., O. galioides (DC.) R.M. King \& H. Rob., O. hartwegii (B.L. Rob.) R.M. King \& H. Rob., O. hoppii (B.L. Rob.) R.M. King \& H. Rob., O. macbridei B.L. Rob., O. mathewsii (B.L. Rob.) R. M. King \& H. Rob., O. ovatus B.L. Rob., O. pubescens (Sm.) R.M. King \& H. Rob. y O. sagasteguii H. Rob. (Brako \& Zarucchi, 1993; Ulloa Ulloa et al., 2004; Beltrán et al., 2006). Las últimas especies que fueron descritas para Perú fueron $O$. ferreyrii y O. sagasteguii (Robinson, 1998).

Revisando críticamente tanto el material de herbario como el de las últimas colecciones procedentes de los andes peruanos, en particular del Norte con énfasis en Cajamarca (Prov. Contumazá), se ha encontrado una nueva especie, cuya descripción, ilustración y discusión con sus relacionados es el objetivo de este trabajo.

\section{Material y métodos}

El estudio está basado en la revisión de material de las colecciones presentes en los herbarios F, HAO, HUT, MO; y observaciones directas de hábito y hábitat en el campo, efectuadas en varios viajes realizados en los años de 1977, 1991 y 2006 a diversas localidades del Distrito de Guzmango, Provincia Contumazá, Departamento de Cajamarca; entre los 2200-2500 m de altitud.

Las colecciones se realizaron de acuerdo con la metodología y técnicas convencionales de herborización. Adicionalmente al trabajo de campo se fijó y conservó material en líquido (alcohol etílico al 70\% o AFA) para estudiar la estructura floral. El material botánico del tipo fue depositado en los siguientes herbarios: F, HUT, MO, US y USM. Son presentadas, la descripción, discusión, delineación y mediciones de la especie (Fig. 1A-E). También se adicionan datos de su ecología y distribución geográ- fica en la zona de Amotape-Huancabamba de elevada riqueza endémica (Weigend, 2002). Los acrónimos de los herbarios son citados según Holmgren et al. (1990).

\section{Taxonomía}

Ophryosporus marchii Sagást. \& E. Rodr. sp.nov.

(Fig. 1 A-E)

Tipo: PERÚ. Dpto. Cajamarca, Provincia Contumazá, alrededores del Distrito de Guzmango, 2500 m, 25 Julio 2006, A. Sagástegui A. 17611 (Holótipo: HUT; Isótipos: F, HUT, MO, US, USM)

Suffrutex 1,50 m altus, caulibus teretibus, glabrescentibus, compactus, 0,6 mm crassus. Folia opposita, petiolata (petiolis dense puberulis 1,5-5 cm longis), submembranacea, ovata vel oblongo-eliptica, glabrescentia, basi asimetrica, apice acuminata, irregulariter dentato-mucronulata, 3-nervata, 7-12 cm longa et 3-6 cm lata. Capitulescentia thyrsoidea. Capitula sessilia 4-flora, 6-7 mm alta et 1-1,5 mm crassa; bracteis involucralibus 4(-5), 1-seriatis, oblongis, subacutis, glabrescentibus, 3-4 mm longis et 0,5-0,8 $\mathrm{mm}$ latis; corolla tubulata, alba, 5-lobulata, lobulis triangularis $0,5 \mathrm{~mm}$ longis. Achaenia (inmatura) atrobrunnia, glabra, 2-2,5 mm longa, 5-costata; carpopodium breve; pappus nullis.

Sufrútice de $1,50 \mathrm{~m}$ de alto, tallos quebradizos, teretes, marrones, lisos, glabrescentes (ligeramente pubérulos cerca de los nudos), compactos (medulosos), hasta 0,6 mm de diámetro, con ramificaciones opuestas. Hojas opuestas, pecioladas (pecíolos acanalados, densamente pubérulos, $1,5-5 \mathrm{~cm}$ de largo), submembranáceas; limbos desde ovados hasta oblongo-elípticos, asimétricos en la base, acuminados en el ápice, irregularmente dentado-mucronulados, trinervados desde cerca de la base, glabrescentes, los mas grandes $7-12 \mathrm{~cm}$ de largo por 3-6 cm de ancho. Capitulescencia tirsoide, de hasta $65 \mathrm{~cm}$ de largo, ramificaciones laterales $2(-3)$ por nudo, dispuestas sobre un eje central, divididas dicotómicamente en el ápice, pedúnculos 
|a
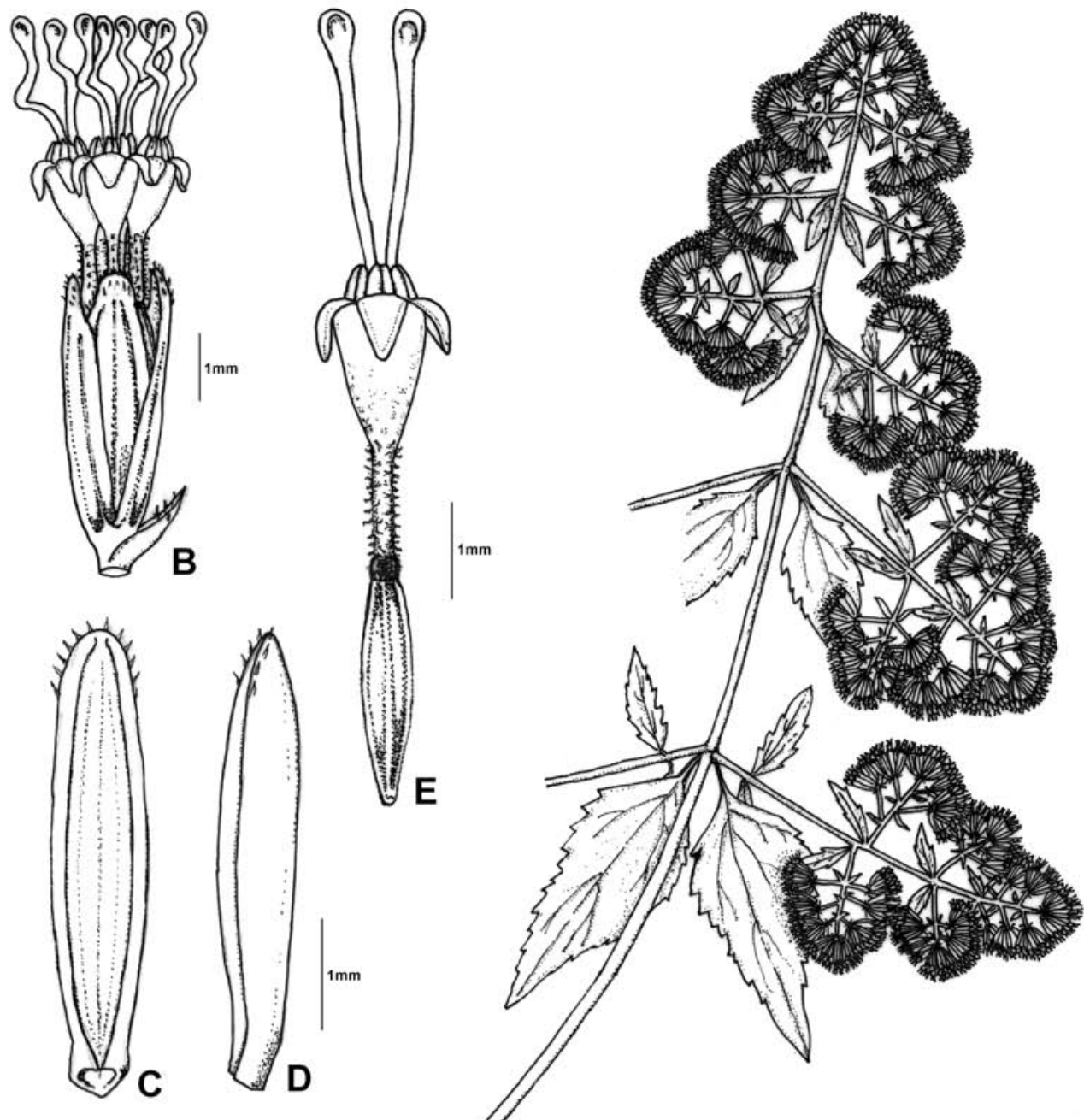
florales $2-3 \mathrm{~mm}$, con $4-6$ capítulos reunidos en forma de glomérulos; densamente pubérulos. Capítulos sésiles, 4- floros, 6-7 $\mathrm{mm}$ alto, $1-1,5 \mathrm{~mm}$ de diámetro; brácteas involucrales $4(-5)$ en una serie, glabrescentes, oblongas, $3-4 \mathrm{~mm}$ largo, $0,5-0,8 \mathrm{~mm}$ ancho, ápice subagudo; corola blanca, pubérula, $2-2,5 \mathrm{~mm}$ de largo, tubulosa, tubo cilíndrico, $1-1,5 \mathrm{~mm}$ de longitud, $1 \mathrm{~mm}$ de ancho en la garganta, 5-lobulada, lóbulos triangulares, 0,5 mm de largo. Estambres 5, ligeramente exertos, anteras $1 \mathrm{~mm}$; ramas estigmáticas bifurcadas, largamente exertas, $4-5 \mathrm{~mm}$ de largo. Cipselas (inmaduras), $2-2,5 \mathrm{~mm}$ de largo, 5-costado, fusiformes, atroparduscas, glabras; carpopodio corto; papus ausente.

\section{Material adicional examinado:}

PERÚ: Dpto. Cajamarca, Provincia Contumazá: Arriba de Trinidad, 2200 m, 6 Julio 1977, A. Sagástegui A., E. Alvitez I. \&J. Mostacero L. 8954 (HUT, MO); Travesía de Shundón (alrededores de Guzmango), 2500 m, 24 Julio 1991, A. Sagástegui A. 14449 (HAO, F).

Discusión Taxonómica: Afín a $O$. sagasteguii H. Rob. de la misma área de estudio (ver colección tipo: A. Sagástegui A. et al. 9975; citada por Robinson, 1998); sin embargo se diferencia principalmente porque la nueva especie presenta tallos compactos (vs. fistulosos), por la forma y tamaño de las hojas y capítulos, 4 flores por capítulo (vs. 5 flores) y por carecer de papus.

Distribución y Ecología: Se conoce de la localidad de donde procede el tipo y sus alrededores, por lo tanto es aparentemente endémica, creciendo entre 2200 y $2500 \mathrm{~m}$ de altitud en suelos negros de humificación variable; perteneciente a la denominada zona de Amotape-Huancabamba de elevada riqueza endémica. Asociada con Baccharis sp. (Asteraceae), Boerhavia sp. (Nyctaginaceae) y Paracalia jungioides (Hooker \& Arnott) Cuatrecasas (Asteraceae), y con quienes vegetan, generalmente a lo largo de los cercos de las chacras y bosques secundarios.

Etimología: Es un honor dedicar esta nueva especie al Señor don Juan March Ordinas (Español), por su permanente apoyo al desarrollo de las actividades científicas y sociales a través del Instituto Juan March de Estudios e Investigaciones.

Estado de Conservación: Utilizando los criterios de la Lista Roja UICN (UICN, 2001), esta especie endémica debería ser incluida en la categoría VU (Vulnerable), debido a que el área de presencia y ocupación es pequeña y sin protección por el Estado; así mismo, por el reducido número y tamaño de sus poblaciones que actualmente se encuentran afectadas por la ampliación de la frontera agrícola.

\section{Agradecimientos}

Se agradece a los directores y curadores de los herbarios mencionados en el trabajo por facilitar el ingreso a sus instituciones durante nuestras visitas o brindar datos de sus colecciones. A la Dra. Blanca León (TEX, USM) por proveer literatura especializada e imágen del holótipo de Ophryosporus sagasteguii $\mathrm{H}$. Rob. depositado en US. A Sandra Arroyo Alfaro (HUT) por la preparación de la ilustración de la especie que es objeto del presente estudio.

\section{Literatura citada}

Brako, L. \& J. Zarucchi. 1993. Catálogo de las Angiospermas y Gimnospermas del Perú. Monogr. Syst. Bot. Missouri Bot. Garden. 45: 159

Beltrán, H.; A. Granda; B. León; A. Sagástegui; I. Sánchez \& M. Zapata. 2006. Asteraceae endémicas del Perú. En El libro rojo de las plantas endémicas del Perú. Ed.: Blanca León et al. Rev. peru. biol. Número especial 13(2): 64-164.

Holmgren, P. K.; N. H. Holmgren \& L. C. Barnett. 1990. Index Herbariorum. Part. I: The Herbaria of the World. $8^{\text {th }}$. ed. The New York Botanical Garden, Bronx, New York. U.S.A.

Robinson, H. 1998. New species and new combinations of neotropical Eupatorieae (Asteraceae). Phytologia. 84(5): 347-353.

UICN. 2001. Categorías y Criterios de la Lista Roja de la UICN. Versión 3.1. Preparado por la Comisión de Supervivencia de Especies de la UICN. UICN, Gland, Suiza y Cambridge, Reino Unido. ii $+33 p p$.

Ulloa Ulloa, C.; J. L. Zarucchi \& B. León. 2004. Diez años de adiciones a la Flora del Perú: 1993-2003. Arnaldoa (Edic. Espec., Nov. 2004). Pág. 36.

Weigend, M. 2002. Observations on the Biogeography of the Amotape-Huancabamba Zone in Northern Peru. In: K. Young et al., Plant Evolution and Endemism in Andean South America. Bot. Review 68(1): 38-54. 


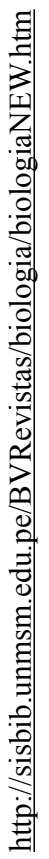

